\title{
Capacitive Fill-Level Measurement Using Configurable Electrodes for Adaptivity
}

\author{
Christoph Kandlbinder ${ }^{1}$, Teresa Sporer ${ }^{1}$, Tobias Siegel ${ }^{1}$, Patrick Mößle ${ }^{1}$, \\ Alice Fischerauer ${ }^{1}$, Tristan Zürl'1 , Gerhard Fischerauer ${ }^{1}$ \\ ${ }^{1}$ Chair of Measurement and Control Systems, University of Bayreuth, 95440 Bayreuth, Germany, \\ mrt@uni-bayreuth.de
}

\begin{abstract}
:
The observation of fluids and their properties is a frequent task in the field of process engineering. As capacitive measurement techniques can well distinguish between higher-permittivity liquids such as water on the one hand and gases such as air on the other hand, they are well suited to determine liquid/gas distributions. One such application is the contactless measurement of water fill level in a pipe. We designed, implemented and tested a capacitive measurement system for this application. It consists of electrodes distributed along the pipe circumference which can be combined electronically to form smaller or larger "synthetic electrodes". By measuring the capacitances between the electrodes, one obtains information about the water/gas distribution inside the pipe with a spatial resolution depending on the size of the configurable electrodes. Thus, it becomes possible to quickly obtain coarse fill-level information with larger electrodes and then refine the spatial resolution in a region of interest with a small-electrode configuration (which incurs longer measurement times). The system was successfully tested on a test bench with a pipe inside of which the liquid could be made to slosh by axial movements.
\end{abstract}

Key words: capacitance, adaptivity, electrodes, configurable, fill-level.

\section{Introduction}

For processes in which fluids or solid particles are transported in pipes, it is of crucial interest to investigate the fill level or even the distribution of granular solids in particle flows [1]. The literature reports on measurements for liquids like water inside cuboids [2], on special design considerations for the electrode configuration [3], on coupled-field problems (fluid field and electrical field) [4], on the analysis of two-phase slug flows based on neural networks [5], and many other aspects.

The advantages of capacitive sensing are that electrodes can be constructed in a non-invasive and non-intrusive manner around a volume to be investigated. This is, e. g., used in electrical capacitance tomography (ECT). If electrodes are placed around a non-conducting pipe, it is possible to measure differences in the resulting capacitance when the permittivity changes in the space between two electrodes. With fluid or solid particles having a permittivity different from that of the surrounding gas, a change in fill level leads to a change in permittivity between electrodes. By using multi-electrodes and reconstruction techniques, the spatial distribution of the fluid can be determined [6].
The spatial resolution for the permittivity is influenced by the number and the geometry of the electrodes used. The bigger an electrode, the larger the capacitance associated with it and the better the signal-to-noise ratio (SNR). The smaller an electrode, the smaller the SNR. On the opposite side, with more and smaller electrodes around the probed volume, the spatial resolution improves at the cost of measurement speed because more capacitances need to be measured [7].

Quick and coarse measurements on the one hand and slow and fine measurements on the other hand are enabled by "synthetic electrodes" which are electronically combined from elementary electrodes and which can be made to have various sizes and shapes. Such configurable electrodes have already been proposed by [8] and later by [9] and [10]. Ref. [10] describes a concept of adaptivity in which the combined electrodes are excited by voltages of different amplitudes and phases to optimize the electrical field distribution.

We propose another approach to adaptivity in our present contribution. It is to cope with the unwanted side-effect of configurable electrodes, viz., the high number of possible electrode 
combinations. The number of measurements must be restricted unless a high calculation complexity and small frame rates are acceptable. By way of example, we have studied the problem of fill level measurement in a pipe and have investigated how to operate configurable electrodes so that the number of measurements remains acceptable and that the spatial resolution in regions of interest suffices. We fitted a pipe on a test bench with a capacitive fill-level measurement system to experimentally demonstrate the approach.

\section{Test bench}

The test bench was used to make fluids or granular fillings inside a test pipe slosh. Fig. 1a) shows the test pipe comprising two coaxial acrylic glass pipes to allow visual control of its interior. Between the two acrylic pipes, polycarbonate rings carrying copper electrodes either on the inside or on the outside of the ring were inserted. An example of such an electrode ring is shown in Fig. 1b) together with its electrode numbering in Fig. 1c).

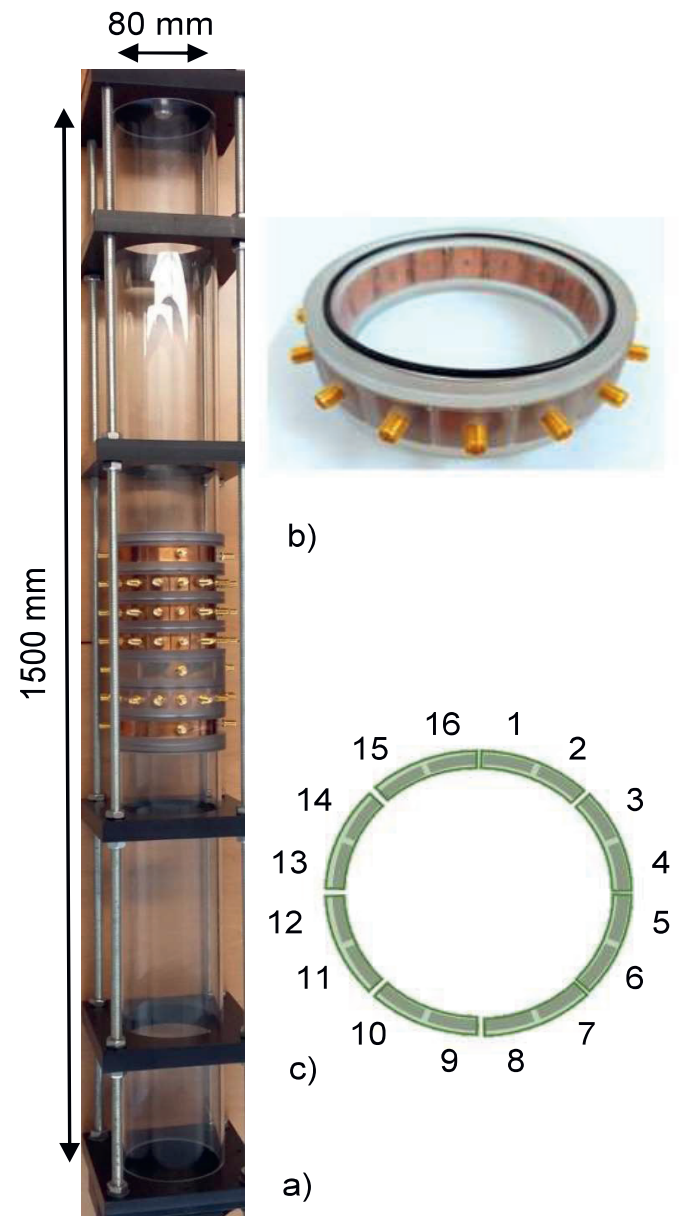

Fig. 1. Test-bench details. a) Pipe (shown upright here, but mounted horizontally on the test bench). b) Electrode ring with electrodes on the outside of the wall. c) Numbering of the electrodes.
To induce a periodic sloshing of the fluid or the granular filling inside the pipe, the test bench was made to carry out axial movements by a compressed-air actuator. The maximum amplitude of the oscillations was $30 \mathrm{~cm}$. The velocity and amplitude of the oscillations was controlled by a computer via Labview.

\section{Capacitance measurement}

Our capacitance measurement system consists of a capacitance-to-digital converting IC (Acam PCap01), an analog switch matrix, a microcontroller for acquisition and controlling (TIVA DK-TM4C123G), and a computer for data processing (Fig. 2).

The switch matrix comprises 16 single-pole quadruple-throw, or SP4T, analog switches (TI TS5A3359). The single pole of each switch is connected to a single electrode. By the switching action, the electrode can either be connected to ground or to high impedance (open circuit) or to port1 or to port 2 of the capacitance measurement IC. The IC measures the capacitance by determining the discharge time of the capacitance, inserted between ports 1 and 2 of the IC, via a resistor. The time is then compared to the discharge time of a known reference capacitance via the same resistor [11]. The digital capacitance information is transferred to the microcontroller, which controls the switches, sends commands to the capacitance measurement IC, and transfers the measured data to the digital computer serving to evaluate the data.

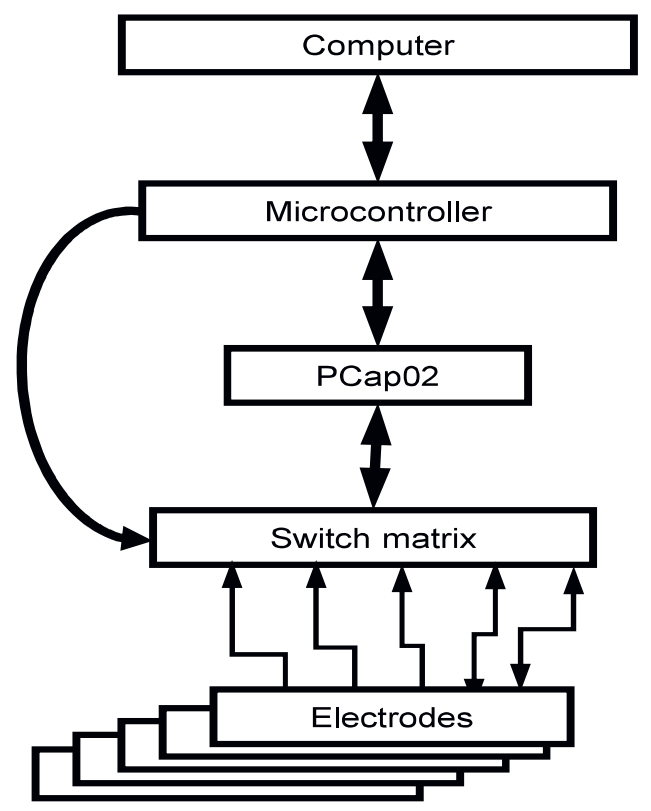

Fig. 2. Capacitance measurement system 


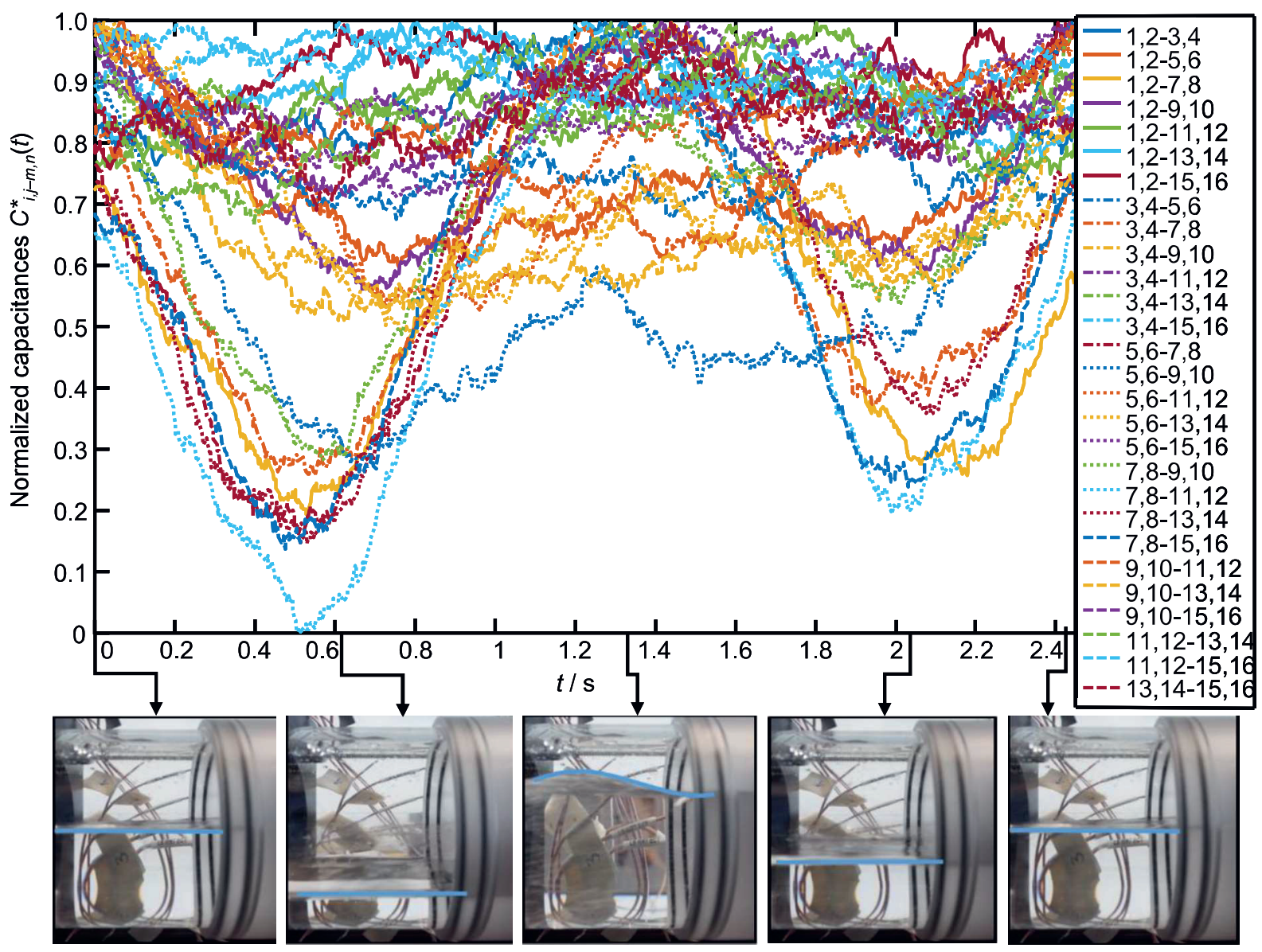

Fig. 3. Normalized capacitances $C_{i, j-m, n}^{*}(t)$ for 28 electrode combinations (electrodes numbered as in Fig. 1c) over one sloshing period. The photos show the water surface inside the pipe at selected instants.

Using the SP4T switches, one can easily combine electrodes by simultaneously connecting them to the same port of the capacitance measurement IC. The other electrodes can either be connected to the other IC port, or they are grounded or left floating (open-circuit case). This has different influences on the measured values as shown in [12]. The "floating" mode yields a higher SNR and has therefore been used in our investigations.

\section{Results}

With $M$ electrodes, one can perform $k=0,5 \cdot M$. $(M-1)$ linearly independent measurements; our 16-electrode ring allows 120 linearly independent measurements. If electrodes are combined, they act as bigger electrodes. For instance, an 8-electrode ring is obtained if adjacent electrodes are combined pairwise. This configuration then allows $k=28$ linearly independent measurements.

Fig. 3 shows the normalized time-variant capacitances $C_{i, j-m, n}^{*}(t)$ between various electrode pairs $(i, j)$ and $(m, n)$ when the pipe on the test bench was partially filled with water and the water was made to slosh. The pipe movement was periodic with a time period of $T_{\mathrm{p}}=3,035 \mathrm{~s}$. The measurement time for the 28 capacitances (based on electrode rings on the outside) amounted to $T_{\text {meas }} \approx 883 \mathrm{~ms}$. Hence, every capacitance was measured about 3.4 times per full sloshing cycle. Fig. 3 resulted from a mapping of the data recorded during a total measurement time of 30 minutes to the time interval $\left[0, T_{\mathrm{p}}\right]$ by assuming periodic capacitance changes: $C_{i, j-m, n}(t)=C_{i, j-m, n}\left(t-\ell \cdot T_{\mathrm{p}}\right)$ with $\ell$ integer. To suppress noise, a moving-average filter of length 200 was applied to the data.

As can be seen in Fig. 3, only some electrode combinations allow one to estimate the water fill level. Others, associated with electric field lines that do not run through the air-water interface, hardly show any response.

The electrode combinations lead to different base capacitances. Therefore, to render the measured capacitances during sloshing more comparable, Fig. 3 shows normalized capacitances $C_{i, j-m, n}^{*}(t)$. This $C_{i, j-m, n}^{*}(t)$ is obtained from the deviation of the capacitance $C_{i, j-m, n}(t)$ from its maximum value at any one time, 
$\Delta C_{i, j-m, n}(t)=C_{i, j-m, n}(t)-\max \left\{C_{i, j-m, n}(t)\right\}<0, \quad$ by $C_{i, j-m, n}^{*}(t)=1+\Delta C_{i, j-m, n}(t) / \Delta C_{\max }$. Here, $\Delta C_{\max }=$ $\max \left\{-\Delta C_{7,8-11,12}(t)\right\}$ is the largest capacitance swing observed for any electrode configuration.

To identify the electrode pairs with capacitances that contain the most usable fill-level information, the maximum normalized capacitance swings during one sloshing period are shown in a bar plot in Fig. 4. The configurations with the largest capacitance swings comprise adjacent electrode pairs. These pairs respond most sensitively to permittivity changes in the near-wall region between the electrode pairs. The pipe wall strongly influences the capacitance between such electrodes as $\varepsilon$ r, polycarbonate $=2.9 \quad<\quad \varepsilon r$, water $=80 \quad$ [3]. Such adjacent-electrode capacitances are neglected in the further signal processing.

In a next step, capacitances of all electrode combinations with swing of at least, say, $30 \%$ are retained as this change can be separated from measurement noise and correlates well with the fill level. The associated electrode-pair combinations of the synthetic 8-electrode system are then linked to elementary-electrode combinations of the 16-electrode system. That is, the electrode pairs are split into their respective individual electrodes and the capacitances between any of the individual electrodes are measured (e. g., the combination $7,8-11,12$ in the 8 -electrode system is linked to the four combinations $7-11,7-12,8-11$, and 812 in the 16-electrode system).
Fig. 6 demonstrates that the approach correctly singles out the elementary-electrode combinations in the 16-electrode system which are associated with the largest capacitance swings and therefore the largest sensitivities to fill-level changes. The bar plot shows the capacitance swings of all 120 possible electrode combinations with the 74 combinations preferred according to the results of the 8-electrode measurement in red and the adjacent-electrode combinations to be avoided in green. 60 out of the 74 largest capacitance swings belong to preferred capacitances. Hence, the latter clearly mirror the sloshing movement.

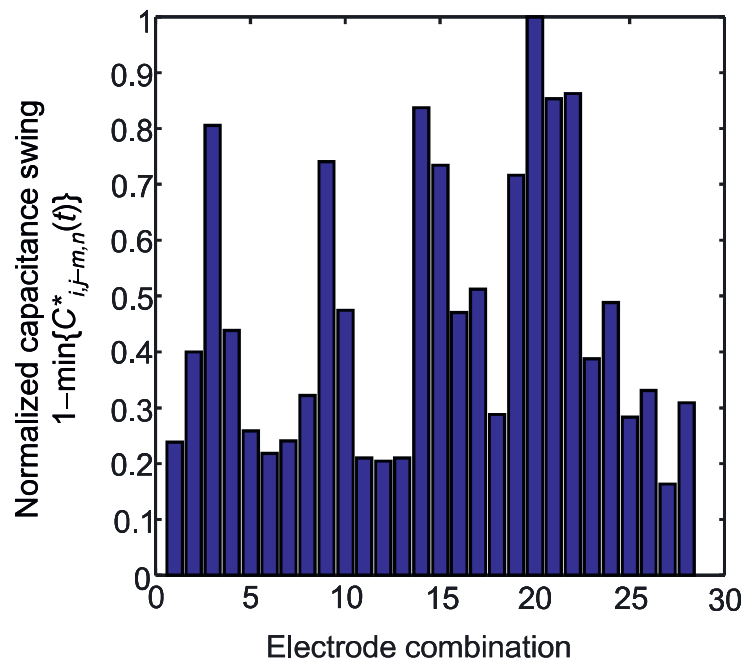

Fig. 4. Maximum change of measured normalized capacitances during one sloshing period for the 28 electrode combinations also used for Fig. 3.

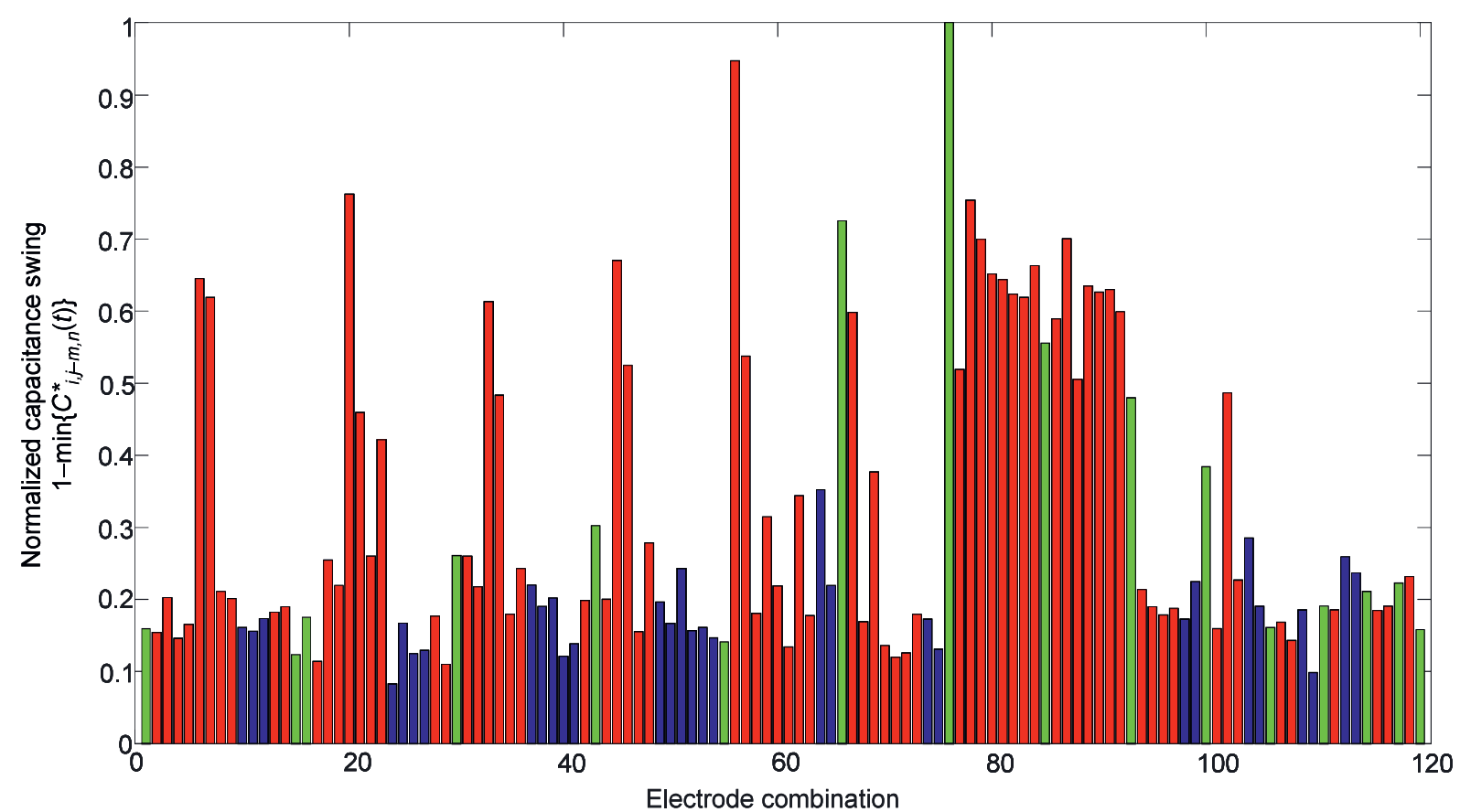

Fig.6. Normalized capacitance swings for the 120 electrode combinations possible with 16 elementary electrodes. The combinations to be measured according to the results of an 8-electrode measurement are marked in red, and neglected adjacent-electrode-pair cases are marked in green. 


\section{Conclusion}

A 16-electrode configuration, by which the filllevel of a pipe or the sloshing movement of water inside the pipe may be monitored capacitively, requires the measurement of $k=120$ capacitances $C_{i-j}$ between the electrodes to obtain full information. The computational effort for this is considerable. We have shown that it suffices to measure $k=28+74=102$ capacitances at most to obtain the same information. To be more specific, one needs to measure the 28 capacitances between pairs of electrodes and then the 74 capacitances between elementary electrodes selected according to the results of the previous 28 measurements.

In practice, the system will be especially advantageous when the processes observed affect only few capacitances. In the case considered, a subset of the preferred 74 capacitances will be entirely sufficient. This then requires an algorithm which selects the subset most affected by permittivity changes inside a region of interest.

In future work, the adaptive measurements are to be evaluated by suitable ECT reconstruction algorithms to generate pictures of the permittivity distribution inside the pipe. Such applications go beyond mere fill-level measurements, which could work with one or very few capacitances.

\section{References}

[1] K. Zhu et al., "Electrical capacitance tomography measurements on vertical and inclined pneumatic conveying of granular solids," Chem. Eng. Sci., Vol. 58, pp. 4225-4245, July 2003, doi: 10.0.3.248/S0009-2509(03)00306-3.

[2] M. A. Nurge, "Electrical capacitance volume tomography with high contrast dielectrics using a cuboid sensor geometry," Meas. Sci. Technol., Vol. 18, No. 5, pp.1511-1520, March 2007

[3] A. J. Jaworski and G. T. Bolton, "The design of an electrical capacitance tomography sensor for use with media of high dielectric permittivity," Meas. Sci. Technol., Vol. 11, pp.743-757, March 2000.

[4] J. Ye et al., "Coupling of fluid field and electrostatic field for electrical capacitance tomography," IEEE Trans. Instrum. Meas., Vol. 64, No. 12, pp. 3334-3353, Dec. 2015.

[5] C. Cozin et al., "Two-phase slug flow characterization using artificial neural networks," IEEE Trans. Instrum. Meas., Vol. 65, No. 3, pp. 494501, March 2016.

[6] F. Wang et al., "Electrical capacitance tomography: Design and Applications," Sensors 2010, Vol. 10, pp. 1890-1917, March 2010.
[7] W. Yang, "Design of electrical capacitance tomography sensors," Meas. Sci Technol., Vol. 21, No. 4, (13pp), Feb. 2010.

[8] N. Reinecke and D. Mewes, "Resolution enhancement for multi-electrode capacitance sensors," in: M. S. Beck: Process tomography - a strategy for industrial exploitation [Proceedings of a workshop sponsored by the Commission of the European Community, Directorate-General for Science, Research and Development, under the Brite/Euram Industrial Technologies R\&D programme (1989-1992), held in Oporto, 24-26 March, 1994]. Manchester: UMIST Dept. of Electrical Engineering and Electronics, 1994, pp. 50-61.

[9] Y. Yang and L. Peng, "A configurable electrical capacitance tomography system using a combining electrode strategy," Meas. Sci Technol., Vol. 24, No. 7, (11pp), June 2013.

[10] Q. Marashdeh et al., "Adaptive electrical capacitance volume tomography," IEEE Sensors J., Vol. 14, No. 4, Apr. 2013.

[11] Acam Messelectronic, Data Sheet "PCap02A Single-chip Solution for Capacitance Measurement," Vol. 1, Vers. 1.6, May 29, 2014.

[12] N. Reinecke and D. Mewes, "Recent developments and industrial/research applications of capacitance tomography," Meas. Sci Technol., Vol. 7, pp. 233-246, Jan. 1996. 